\title{
Soft tissue
}

\section{Oral tumours: basic}

\section{Nicholas Bacon}

This lecture will cover the variety of presentations of oral tumours seen in general practice. It will be designed to be as practical as possible, setting up the background of many oral tumours, which can then be followed by some of the more complex issues and procedures encountered in the second, 'advanced' lecture. The background will include both benign lesions (e.g. epuli, ameloblastomas, hyperplasia, etc.) and malignant oral tumours (e.g melanoma, osteosarcoma, fibrosarcoma, squamous cell carcinoma, multilobular tumour of bone). It will include the bony structures of the mandible, maxilla, hard palate and ventral orbit, plus the soft tissue of the lips, gingiva, tongue, sublingual and salivary tissues, tonsils and soft palate. This lecture will consider common presenting signs, differential diagnoses, biopsy techniques, when to use radiographs and when computed tomography might be helpful, tumour staging, and decision making regarding common oral tumours in cats and dogs. The lecture will follow the tissue of origin, as that is how they present clinically, to improve logical thinking and decision making.

Once a diagnosis has been reached, staging has been performed if appropriate (i.e. what is it, where is it), we will move onto the basic procedures to treat oral tumours (i.e. what to do with it). This will include easyto-follow descriptions and videos of rostral mandibulectomies, rostral maxillectomies, sublingual surgeries, simple lip resections and reconstructions. We will also cover the necessary surgical instrumentation, surgical tips and complications that might be encountered in the perioperative and postoperative periods in both cats and dogs.

The factors that influence prognosis will be covered. A key message of this lecture is how there are always three conflicting forces when considering oral surgery in cats and dogs; oncological outcome, cosmetic outcome and functional outcome. How they interplay and influence each other will be constantly reinforced throughout this and the 'advanced' lecture. Considerate and sound decision making is a key learning message of this lecture.

\section{KEY LEARNING OBJECTIVES}

- Learn the presenting signs, differential diagnoses and investigation of rostral or superficial lesions of the jaw and oral soft tissues

- Understand the surgical technique for biopsy and resection of rostral tumours

- Understand postoperative function, complications and prognosis for described procedures

\section{MULTIPLE CHOICE QUESTIONS}

1. Which of the following are the three most common oral malignancies in cats?

(A) Lymphoma, squamous cell carcinoma, melanoma

(B) Lymphoma, squamous cell carcinoma, osteosarcoma

(C) Squamous cell carcinoma, osteosarcoma, fibrosarcoma

(D) Squamous cell carcinoma, osteosarcoma, melanoma

2. Approximately what percentage of cats will never voluntarily eat again after mandibular surgery?
(A) $6 \%$
(C) $18 \%$
(B) $12 \%$
(D) $24 \%$

3. Which is the first lymphocentre in the head through which all lymphatic drainage passes?
(A) Medial retrophayryngeal lymph node
(B) Lateral retropharyngeal lymph node
(C) Submandibular lymph node
(D) Caudal cervical lymph node

\section{Oral tumours: advanced}

\section{Nicholas Bacon}

The preceding basic lecture set the background surrounding common benign and malignant tumours. This advanced lecture is more focused on the complex presentation of more caudally based tumours, more advanced disease, and how the intricate anatomy of the caudal skull impacts surgical planning. Cross-sectional imaging plays a more significant role now in planning caudal mandibulectomies (horizontal body, vertical ramus, hemimandibulectomy), caudal maxillectomies (intraoral, combined maxillectomy-orbitectomy) and glossectomies. The necessary surgical instrumentation will be covered, and step-by-step approaches to some of the larger surgeries demonstrated through images and videos.

The differences between oral surgery in cats and dogs will be emphasized, and how this impacts morbidity and prognosis. A key emphasis will be on the relatively common dysphagia seen in the immediate postoperative period in cats.

The steps involved in removing the submandibular and medial retropharyngeal lymph nodes will also be covered as nodal staging is a key aspect of understanding the tumour biology in the patient.

More complex oral surgery is also associated with larger and more significant complications, not limited to bleeding, pain, dehiscence, local recurrence (often due to incomplete surgical margins through poor surgical planning or execution) and altered function. Necessary surgery to address dehiscence and oronasal fistulae will be covered.

Finally adjuvant therapies for oral malignancies in cats and dogs will be introduced. This includes awareness 
of appropriate chemotherapy agents, possible immunotherapy agents and when external beam radiation is indicated. Appropriate protocols, agents and toxicities will be covered.

\section{KEY LEARNING OBJECTIVES}

- Be aware of the types and indications for complex mandibular surgery in cats and dogs

- Be aware of the types and indications for complex maxillary surgery in cats and dogs

- Understand the adjuvant therapies for common malignancies in cats and dogs

\section{MULTIPLE CHOICE QUESTIONS}

1. Which of the following is a key feature of decision making when planning mandibular resection?

(A) The number of teeth involved
(B) Whether the tumour has entered the mandibular alveolar canal

(C) If the tumour crosses midline

(D) If the overlying skin is invaded

2. What does an appropriate choice for adjuvant chemotherapy for tonsillar squamous cell carcinoma include?
(A) Vinblastine
(B) Lomustine
(C) Cyclophosphamide
(D) Carboplatin

3. For osteosarcoma affecting the oral cavity, what is one of the most important prognostic factors?

(A) Mitotic index (per 10 high powered fields)

(B) A clean surgical margin

(C) Draining lymph nodes free of metastasis

(D) Degree of bone destruction

\section{Mapping lymph nodes: the whys and the wherefores}

\section{Nicholas Bacon}

The detection of lymph node metastasis is important to quantify spread of cancer in cats and dogs. Although the majority of sarcomas disseminate by vascular invasion, carcinomas and round cell tumours primarily spread through the lymphatic system. Determining exactly which is the draining lymph node to these primaries, however, might require sentinel lymph node mapping. Very often the regional lymph node is the sentinel lymph node, that is, the one you might anticipate, but sometimes nodes in unpredictable anatomical locations might also function as the sentinel node.

Accurate nodal staging might rely on palpation, fine needle aspiration, or even lymph node extirpation, but all rely on the node being sampled to be the sentinel node. In small animal practice a variety of techniques have been described for mapping, including radiographic lymphangiography, intraoperative blue dye mapping, computed tomography (CT) lymphangiography, contrast-enhanced ultrasonography and lymphoscintigraphy.

Sentinel lymph node mapping in veterinary medicine needs to be simple, inexpensive, accurate and safe, if it is to have a significant chance of becoming more mainstream. There must be a short learning curve, with minimal additional equipment being required. Techniques which fit into this category, including their benefits and drawbacks, will be covered in the lecture.

\section{KEY LEARNING OBJECTIVES}

- Understand that predicting which lymph node will drain a particular tumour is not straightforward

- Be aware that identifying the sentinel (first) lymph node is important for accurate and appropriate staging

- Know what techniques exist that can be employed in daily practice to understand tumour biology of a specific patient

\section{MULTIPLE CHOICE QUESTIONS}

1. Which of the following is correct about sentinel lymph node mapping?
(A) Identifies only positive metastatic lymph nodes
(B) Identifies the first draining lymph node
(C) Can only be performed at the time of surgery
(D) Will be successful in $100 \%$ cases

2. Which of the following should be done in CT lymphangiography?
(A) The contrast should be injected directly into the tumour
(B) The contrast should be injected without dilution
(C) The tumour tissue should be massaged after injection to promote drainage
(D) Contrast should only be injected peritumourally

3. What should the dilution of iodine contrast solution be for CT lymphagiography?
(A) $20 \%$ dilution
(C) $33 \%$ dilution
(B) $25 \%$ dilution
(D) $50 \%$ dilution 